\title{
Blow-Up of Solution to Cauchy Problem for the Singularly Perturbed Sixth-Order Boussinesq-Type Equation
}

\author{
Changming Song, Li Chen \\ College of Science, Zhongyuan University of Technology, Zhengzhou, China \\ Email: cmsongh@163.com
}

Received 6 May 2015; accepted 7 July 2015; published 14 July 2015

\begin{abstract}
We consider the singularly perturbed sixth-order Boussinesq-type equation, which describes the bidirectional propagation of small amplitude and long capillary gravity waves on the surface of shallow water for bond number (surface tension parameter) less than but very close to $1 / 3$. The sufficient conditions of blow-up of solution to the Cauchy problem for this equation are given.
\end{abstract}

\section{Keywords}

Singularly Perturbed Sixth-Order Boussinesq Equation, Cauchy Problem, Blow-Up of Solution

\section{Introduction}

In this paper, we consider the following Cauchy problem

$$
\begin{gathered}
u_{t t}=u_{x x}+\sigma(u)_{x x}+\alpha u_{x^{4}}+\beta u_{x^{6}}, x \in R, t>0, \\
u(x, 0)=u_{0}(x), u_{t}(x, 0)=u_{1}(x), x \in R,
\end{gathered}
$$

where $u(x, t)$ is the unknown function, $\sigma(s)$ is the given function, $\alpha>0$ and $\beta>0$ are real numbers, $u_{0}(x)$ and $u_{1}(x)$ are given initial value functions.

In [1], the author has proved the existence and uniqueness of the global generalized solution and the global classical solution for the initial boundary value problem of Equation (1.1).

In [2], the author has discussed the nonexistence of global solution to the initial boundary value problem of Equation (1.1) in some condition. lem

In order to prove that blow-up of Cauchy problem (1.1), (1.2), we shall consider the following auxiliary prob-

$$
\begin{gathered}
v_{t t}=v_{x x}+\sigma\left(v_{x}\right)_{x}+\alpha v_{x^{4}}+\beta v_{x^{6}}, x \in R, t>0, \\
v(x, 0)=v_{0}(x), v_{t}(x, 0)=v_{1}(x), x \in R .
\end{gathered}
$$

Then, we can obtain blow-up of the Cauchy problem (1.1), (1.2) from (1.3), (1.4) by setting $v_{x}(x, t)=u(x, t)$, 
$v_{0 x}(x)=u_{0}(x)$ and $v_{1 x}(x)=u_{1}(x)$.

\section{Main Theorems}

Throughout this paper, we use the following notation: $\|\cdot\|=\|\cdot\|_{L^{2}(\mathrm{R})}$. Now, we give the following main lemmas and theorems.

Lemma 2.1 (convex lemma [3]) Suppose that a positive twice-differential function $H(t)$ satisfies on $t \geq 0$ the inequality

$$
\ddot{H}(t) H(t)-(1+\beta) \dot{H}(t)^{2} \geq-2 A_{1} H(t) \dot{H}(t)-A_{2} H(t)^{2}, \forall t \in R,
$$

where $\beta>0$ and $A_{1}, A_{2} \geq 0$ are constants, $\cdot=\frac{d}{d t}$.

(1) If $A_{1}=A_{2}=0, H(0)>0$ and $\dot{H}(0)>0$, then there exist a $t_{1} \leq t_{2}=\frac{H(0)}{\beta \dot{H}(0)}$, such that $H(t) \rightarrow \infty$ as $t \rightarrow t_{1}$.

(2) If $A_{1}+A_{2}>0, H(0)>0$ and $\dot{H}(0)>-\gamma_{2} \beta^{-1} H(0)$, then $H(t) \rightarrow \infty$ as $t \rightarrow t_{1} \leq t_{2}$, where $\gamma_{1,2}=-A_{1} \pm \sqrt{A_{1}^{2}+\beta A_{2}}$

and

$$
t_{2}=\frac{1}{2 \sqrt{A_{1}^{2}+\beta A_{2}}} \operatorname{In} \frac{\gamma_{1} H(0)+\beta \dot{H}(0)}{\gamma_{2} H(0)+\beta \dot{H}(0)}
$$

Lemma 2.2 [4] Suppose that $s=m+\frac{1}{2}+\lambda, \lambda \in(0,1), m \in Z_{+}$, then $H^{s}(R)$ may be embedded into $C^{m, \lambda}(R)$, and for any $u \in H^{s}(R)$, we have

$$
\left|D^{\alpha} u(x)\right| \rightarrow 0(|x| \rightarrow \infty), \forall \alpha \in Z_{+},|\alpha| \leq m,
$$

where $Z_{+}$is a set of nonnegative integers.

Lemma 2.3 Suppose that $v_{0} \in H^{3}(R), v_{1} \in L^{2}(R), \sigma \in C^{1}(R), F(s)=\int_{0}^{s} \sigma(\tau) d \tau$ and $F\left(v_{0 x}\right) \in L^{1}(R)$, then the solution $v(x, t)$ of the auxiliary problem (1.3), (1.4) satisfies the following energy identity

$$
E(t)=\left\|v_{t}(\cdot, t)\right\|^{2}+\left\|v_{x}(\cdot, t)\right\|^{2}-\alpha\left\|v_{x^{2}}(\cdot, t)\right\|^{2}+\beta\left\|v_{x^{3}}(\cdot, t)\right\|^{2}+2 \int_{-\infty}^{\infty} F\left(v_{x}\right) d x=E(0) .
$$

Proof Multiplying both sides of (1.3) by $2 v_{t}(x, t)$, integrating on $R$, integrating by parts and lemma 2.2, we get

$$
\frac{d}{d t}\left[\left\|v_{t}(\cdot, t)\right\|^{2}+\left\|v_{x}(\cdot, t)\right\|^{2}-\alpha\left\|v_{x^{2}}(\cdot, t)\right\|^{2}+\beta\left\|v_{x^{3}}(\cdot, t)\right\|^{2}\right]+2 \int_{-\infty}^{\infty} v_{x t} \sigma\left(v_{x}\right) d x=0
$$

integrating the product over $[0, t]$, we get the identity (2.2).

Theorem 2.1 Suppose that $v_{0} \in H^{3}(R), v_{1} \in L^{2}(R), \sigma \in C^{1}(R), F(s)=\int_{0}^{s} \sigma(\tau) d \tau, F\left(v_{0 x}\right) \in L^{1}(R)$, and there exists constant $\gamma>0$ and $C_{1}>0$, such that

$$
s \sigma(s) \leq 2(1+2 \gamma) F(s)+2 \gamma\left(1-C_{1} \alpha\right) s^{2}, \forall s \in R .
$$

Then, the solution $v(x, t)$ of the auxiliary problem (1.3), (1.4) blows-up in finite time if one of the following conditions holds

(1) $E(0)<0$;

(2) $E(0)=0, \int_{-\infty}^{\infty} v_{0}(x) v_{1}(x) d x>0$; 
(3) $E(0)>0, \int_{-\infty}^{\infty} v_{0}(x) v_{1}(x) d x>\sqrt{\frac{1}{2} E(0)\left\|v_{0}\right\|^{2}}$.

Proof Suppose that the maximal time of the solution for (1.3), (1.4) is infinite. Let

$$
H(t)=\|v(\cdot, t)\|^{2}+\beta_{0}\left(t+t_{0}\right)^{2},
$$

where $\beta_{0}$ and $t_{0}$ are undetermined nonnegative constants. Differentiating (2.4) with respect to $t$, we have

$$
\dot{H}(t)=2 \int_{-\infty}^{\infty} v(x, t) v_{t}(x, t) d x+2 \beta_{0}\left(t+t_{0}\right) .
$$

By using the Hölder inequality, it follows from (2.5) that

$$
\dot{H}(t)^{2} \leq 4 H(t)\left[\left\|v_{t}\right\|^{2}+\beta_{0}\right] .
$$

Differentiating (2.5) with respect to $t$, making use of (1.3) and (2.2), we get

$$
\begin{aligned}
\ddot{H}(t)= & 2\left\|v_{t}(\cdot, t)\right\|^{2}+2 \int_{-\infty}^{\infty} v(x, t) v_{t t}(x, t) d x+2 \beta_{0} \\
= & 4(1+\gamma)\left[\left\|v_{t}(\cdot, t)\right\|^{2}+\beta_{0}\right]-(2+4 \gamma)\left[E(0)+\beta_{0}\right]+4 \gamma\left[\left\|v_{x}(\cdot, t)\right\|^{2}-\alpha\left\|v_{x^{2}}(\cdot, t)\right\|^{2}\right. \\
& \left.+\beta\left\|v_{x^{3}}(\cdot, t)\right\|^{2}\right]-2 \int_{-\infty}^{\infty} v_{x} \sigma\left(v_{x}\right) d x+4(1+2 \gamma) \int_{-\infty}^{\infty} F\left(v_{x}\right) d x .
\end{aligned}
$$

By virtue of interpolating inequality,

$$
\left\|v_{x^{2}}(\cdot, t)\right\|^{2} \leq C_{1}\left\|v_{x}(\cdot, t)\right\|^{2}+\frac{\beta}{2 \alpha}\left\|v_{x^{3}}(\cdot, t)\right\|^{2} .
$$

Observing the identity (2.7), we get

$$
\begin{aligned}
\ddot{H}(t) & \geq 4(1+\gamma)\left[\left\|v_{t}(\cdot, t)\right\|^{2}+\beta_{0}\right]-(2+4 \gamma)\left[E(0)+\beta_{0}\right]+4 \gamma\left(1-C_{1} \alpha\right)\left\|v_{x}(\cdot, t)\right\|^{2} \\
& -2 \int_{-\infty}^{\infty} v_{x} \sigma\left(v_{x}\right) d x+4(1+2 \gamma) \int_{-\infty}^{\infty} F\left(v_{x}\right) d x .
\end{aligned}
$$

Combing (2.2), (2.3), (2.4), (2.6) with (2.8), we infer

$$
\ddot{H}(t) H(t)-(1+\gamma) \dot{H}(t)^{2} \geq-(2+4 \gamma)\left[E(0)+\beta_{0}\right] H(t) .
$$

(1) If $E(0)<0$, by taking $\beta_{0}=-E(0)>0$, then

$$
\ddot{H}(t) H(t)-(1+\gamma) \dot{H}(t)^{2} \geq 0 .
$$

When $t_{0}$ is sufficiently large, $\dot{H}(0)>0$. Clearly, $H(0)>0$. It follows from lemma (2.1) that there exists $t_{1} \leq t_{2}=\frac{H(0)}{\gamma \dot{H}(0)}$, such that $H(t) \rightarrow \infty$ as $t \rightarrow t_{1}^{-}$.

(2) If $E(0)=0$, by taking $\beta_{0}=0$, we get

$$
\ddot{H}(t) H(t)-(1+\gamma) \dot{H}(t)^{2} \geq 0 .
$$

By virtue of assumption (2), we see $H(0)>0$ and $\dot{H}(0)>0$. It follows from lemma (2.1) that there exists $t_{1} \leq t_{2}=\frac{H(0)}{\gamma \dot{H}(0)}$, such that $H(t) \rightarrow \infty$ as $t \rightarrow t_{1}^{-}$.

(3) If $E(0)>0$, by taking $\beta_{0}=0$, (2.9) becomes

$$
\ddot{H}(t) H(t)-(1+\gamma) \dot{H}(t)^{2} \geq-(2+4 \gamma) E(0) H(t) .
$$

Defining

$$
J(t)=H^{-\gamma}(t)
$$

then 


$$
\begin{gathered}
\dot{J}(t)=-\gamma H^{-(\gamma+1)}(t) \dot{H}(t), \\
\ddot{J}(t)=-\gamma H^{-(\gamma+2)}(t)\left[\ddot{H}(t) H(t)-(1+\gamma) \dot{H}(t)^{2}\right] \leq \gamma(2+4 \gamma) E(0) H^{-(\gamma+1)}(t) .
\end{gathered}
$$

By virtue of assumption (3), we have $\dot{J}(t)<0$. Let

$$
t^{*}=\sup \{\tau \mid \dot{J}(\tau)<0, \tau \in[0, t)\} .
$$

Thanks to the continuity of $\dot{J}(t), t^{*}$ is a positive number. Multiplying both sides of (2.10) by $2 \dot{J}(t)$, we find

$$
\begin{aligned}
\frac{d}{d t} \dot{J}(t)^{2} & \geq-\gamma^{2}(2+4 \gamma) E(0) H^{-2(\gamma+1)}(t) \dot{H}(t) \\
& =2 \gamma^{2} E(0) \frac{d}{d t} H^{-(2 \gamma+1)}(t), \forall t \in\left[0, t^{*}\right) .
\end{aligned}
$$

Integrating (2.11) with respect to $t$ over $[0, t)$, one gets

$$
\dot{J}(t)^{2} \geq \dot{J}(0)^{2}+2 \gamma^{2} E(0) H^{-(2 \gamma+1)}(t)-2 \gamma^{2} E(0) H^{-(2 \gamma+1)}(0) .
$$

By virtue of assumption (3), we see that

$$
\dot{J}(0)^{2}-2 \gamma^{2} E(0) H^{-(2 \gamma+1)}(0)>0 .
$$

Since $\dot{J}(t)$ is a continuous function, we have for $0 \leq t \leq t^{*}$,

$$
\dot{J}(t) \leq-\left[\dot{J}(0)^{2}-2 \gamma^{2} E(0) H^{-(2 \gamma+1)}(0)\right]^{\frac{1}{2}} .
$$

It follows from the definition of $t^{*}$ that (2.12) holds for all $t \geq 0$. Integrating (2.12) with respect to $t$, we arrive at

$$
J(t) \leq J(0)-\left[\dot{J}(0)^{2}-2 \gamma^{2} E(0) H^{-(2 \gamma+1)}(0)\right]^{\frac{1}{2}} t, \forall t>0 .
$$

Hence there is some $t_{1}$, such that $J\left(t_{1}\right)=0$, where

$$
0<t_{1} \leq t_{2}=J(0)\left[\dot{J}(0)^{2}-2 \gamma^{2} E(0) H^{-(2 \gamma+1)}(0)\right]^{-\frac{1}{2}} .
$$

So $H(t)$ becomes infinite at $t_{1}$.

Thus, $H(t)$ always becomes infinite at $t_{1}$ under the assumption (1) or (2) or (3). This is a contradiction to the fact that the maximal time of existence of the solution is infinite. The theorem is proved.

Theorem 2.2 Suppose that $u_{0} \in H^{2}(R), u_{1} \in L^{2}(R), \sigma \in C^{2}(R), F(s)=\int_{0}^{s} \sigma(\tau) d \tau, F\left(u_{0}\right) \in L^{1}(R)$, and there exist constant $\gamma>0$ and $C_{1}>0$, such that

$$
s \sigma(s) \leq 4(1+2 \gamma) F(s)+4 \gamma\left(1-C_{1} \alpha\right) s^{2}, \forall s \in R .
$$

Then, the solution $u(x, t)$ of the Cauchy problem (1.1), (1.2) blows-up in finite time if one of the following conditions holds

(1) $E_{1}(0)<0$;

(2) $E_{1}(0)=0, \int_{-\infty}^{\infty}\left[\int_{-\infty}^{x} u_{0}(\xi) d \xi\right]\left[\int_{-\infty}^{x} u_{1}(\xi) d \xi\right] d x>0$;

(3) $E_{1}(0)>0, \int_{-\infty}^{\infty}\left[\int_{-\infty}^{x} u_{0}(\xi) d \xi\right]\left[\int_{-\infty}^{x} u_{1}(\xi) d \xi\right] d x>\sqrt{\frac{1}{2} E_{1}(0) \int_{-\infty}^{\infty}\left[\int_{-\infty}^{x} u_{0}(\xi) d \xi\right]^{2} d x}$,

where

$$
E_{1}(t)=\int_{-\infty}^{\infty}\left[\int_{-\infty}^{x} u_{t}(\xi, t) d \xi\right]^{2} d x+\|u(\cdot, t)\|^{2}-\alpha\left\|u_{x}(\cdot, t)\right\|^{2}+\beta\left\|u_{x^{2}}(\cdot, t)\right\|^{2}+2 \int_{-\infty}^{\infty} F(u) d x .
$$

\section{Proof Let}

$$
H_{1}(t)=\int_{-\infty}^{\infty}\left[\int_{-\infty}^{x} u(\xi, t) d \xi\right]^{2} d x+\beta_{0}\left(t+t_{0}\right)^{2}
$$


where $\beta_{0}$ and $t_{0}$ are nonnegative constants as those in Theorem 2.1.

By virtue of assumption Theorem 2.1, $u(x, t)$ satisfies the Equation (1.1) and the initial value condition (1.2) in classical sense. We take the change

$$
u(x, t)=v_{x}(x, t), u_{0}(x)=v_{0 x}(x), u_{1}(x)=v_{1 x}(x),
$$

Then

$$
v(x, t)=\int_{-\infty}^{x} u(\xi, t) d \xi, v_{0}(x)=\int_{-\infty}^{x} u_{0}(\xi) d \xi, v_{1}(x)=\int_{-\infty}^{x} u_{1}(\xi) d \xi .
$$

Substituting the above change (2.13) to the Cauchy problem (1.1), (1.2), we have

$$
\begin{aligned}
& v_{x t t}=v_{x^{3}}+\sigma\left(v_{x}\right)_{x^{2}}+\alpha v_{x^{5}}+\beta v_{x^{7}}, \\
& v_{x}(x, 0)=u_{0}(x), v_{x t}(x, 0)=u_{1}(x) .
\end{aligned}
$$

Integrating (2.14) and (2.15) over $(-\infty, x)$, we obtain

$$
\begin{aligned}
& v_{t t}=v_{x x}+\sigma\left(v_{x}\right)_{x}+\alpha v_{x^{4}}+\beta v_{x^{6}}, \\
& v(x, 0)=v_{0}(x), v_{t}(x, 0)=v_{1}(x) .
\end{aligned}
$$

Let

$$
H(t)=\|v\|^{2}+\beta_{0}\left(t+t_{0}\right)^{2},
$$

where $\beta_{0}$ and $t_{0}$ are nonnegative constants as those in Theorem 2.1. By virtue of assumption Theorem 2.1, the sufficient conditions of blow-up of solution to the Cauchy problem (2.16), (2.17) are fulfilled. Therefore, It follows from theorem 2.1 that $H(t)$ becomes infinite at $t_{1}$ Since by the change (2.13), $H_{1}(t)=H(t)$, so $H_{1}(t)$ becomes infinite at $t_{1}$. Theorem 2.2 is proved.

\section{Fund}

This project is supported by NSF Grant 11271336, NSF of Henan Province Grant 122300410166.

\section{References}

[1] Song, C., Li, H. and Li, J. (2013) Initial Boundary Value Problem for the Singularly Perturbed Boussinesq-Type Equation. Discrete and Continuous Dynamical Systems, 709-717.

[2] Song, C., Li, J. and Gao, R. (2014) Nonexistence of Global Solutions to the Initial Boundary Value Problem for the Singularly Perturbed Sixth-Order Boussinesq Equation. Hindawi Publishing Corporation Journal of Applied Mathematics.

[3] Becken, E.F. and Bellman, R. (1983) Inequalities (Fourth Printing). Springer-Verlag, Berlin.

[4] Y D. (1989) $L^{2}$ Theory of Partial Differential Equations. Peking University Press, Beijing. (In Chinese) 